\title{
A new cytosolic DNA-recognition pathway for DNA-induced inflammatory responses
}

\author{
Jun Meng ${ }^{1}$, Xingguang $\mathrm{Liu}^{2}$ and Xuetao Cao ${ }^{1,2}$ \\ Cellular \& Molecular Immunology (2014) 11, 506-509; doi:10.1038/cmi.2014.57; published online 14 July 2014
}

I nnate recognition of DNA within intracellular compartments or the cytoplasm leads to activation of the immune system, which can result in defense against DNA virus infections or the initiation of pathological responses to self-DNA. In the past several years, the field of DNA sensing has undergone an immense expansion. Many DNA sensors have been proposed, including cGAMP synthase (cGAS), DNA-dependent activator of interferon (IFN)-regulatory factors (also named Z-DNA binding protein 1), absent in melanoma 2 (AIM2), leucine-rich repeat (in Flightless I) interacting protein-1, DExD/H box helicases (DHX9 and DHX36) and IFN-inducible protein IFI16 ${ }^{1-3}$ (Figure 1). The signal transduction pathways that mediate the response to cytosolic DNA have been characterized and are mainly thought to act through the AIM2-dependent secretion of IL-1 $\beta$ or the STING-mediated activation of the TBK1-IRF3 axis, leading to induction of antiviral gene expression, particularly type I IFN ${ }^{1}$ (Figure 1). However, there is little insight on how cytosolic DNA induces activation of the transcription factor $N F-\kappa B$, which is important for the DNA-triggered

\footnotetext{
${ }^{1}$ Institute of Immunology, Zhejiang University School of Medicine, Hangzhou, China and ${ }^{2}$ National Key Laboratory of Medical Immunology \& Institute of Immunology, Second Military Medical University, Shanghai, China

Correspondence: Dr XT Cao, Institute of Immunology, Zhejiang University School of Medicine, 866 Yuhangtang Road, Hangzhou 310058, China.

E-mail: caoxt@immunol.org

Received: 11 June 2014; Accepted: 11 June 2014
}

production of pro-IL-1 $\beta$, TNF and IL-6. Recently, Roth et al. ${ }^{4}$ defined a cytosolic DNA recognition pathway responsible for the DNA-triggered activation of NF- $\kappa \mathrm{B}$ and the induction of pro-IL-1 $\beta$.

The study investigated the role of caspase recruitment domain-containing protein 9 (CARD9) in innate immunity. CARD9 is a non-redundant adaptor protein that functions in the assembly of innate multifunctional signaling complexes. ${ }^{5}$ CARD9 links Syk-coupled C-type lectin receptors to activate inflammatory responses and responds to cytosolic sensors, such as RIG-I and Nod2. ${ }^{6-10}$ By using yeast two-hybrid screening, the authors found an interaction between Rad50 and CARD9, and they confirmed this association in mammalian cells through biophysical techniques and immunoprecipitation of endogenous proteins. Rad50 forms a DNA receptor complex with Mre11 and Nbs1 (the MRN complex), which senses and responds to double-strand breaks of DNA in the nucleus. ${ }^{11}$ Due to the DNA binding ability of Rad50, the authors detected a Rad50dependent link between CARD9 and viral dsDNA. Interestingly, transfection of dendritic cells (DCs) with DNA caused the translocation of Rad50 (and Mre11 and Nbs1) from the nucleus to the cytoplasm. Rad50 was recruited to the dsDNA, and CARD9 was also contained within the dsDNA-Rad50 complex aggregates. The potential function of this complex in DNA-induced innate immunity was then assessed. IL-1 $\beta$ production in response to DNA was impaired not only in CARD9deficient DCs, but also in conditional
Rad50-deficient DCs compared to wildtype cells. In contrast, type I IFN production was unaffected by the deletion of CARD9, whereas STING-deficient DCs showed the opposite results. These findings implicate $\operatorname{Rad} 50$ as an innate immunological sensor of DNA and reveal a novel DNA sensing pathway for IL-1 $\beta$ production.

Next, the authors investigated the mechanisms by which the Rad50-CARD9 complex might mediate the production of IL- $1 \beta$. Consistent with the ability of CARD9 to activate canonical NF- $\kappa B$ signaling for other pathogen-sensing pathways, the authors observed that the translocation of NF- $\mathrm{KB}$ subunits RelA (p65) and c-Rel to the nucleus was defective in CARD9-deficient DCs, specifically after cytosolic transfection of DNA. As it is known that CARD9 engages Bcl-10 to activate NF- $\kappa \mathrm{B}$ in Ctype lectin or RIG-I-activated pathways, the authors investigated the involvement of Bcl-10 during DNA-induced innate immunity in Bcl-10-deficient DCs. They found that $\mathrm{Bcl}-10$ was essential for the activation of NF- $\kappa \mathrm{B}$ and the generation of IL- $1 \beta$, but not for IFN- $\beta$ induced by cytosolic dsDNA. Based on the findings that CARD9 and Bcl-10 acted together to activate NF- $\kappa B$ following cytosolic DNA sensing, the authors demonstrated that Bcl-10 was recruited to the dsDNARad50 complexes in a CARD9-dependent manner. Therefore, Rad50 senses cytosolic DNA and recruits CARD9, which results in the engagement of Bcl10 for the activation of NF- $\kappa B$. This then facilitates the induction of pro-IL-1 $\beta$. 


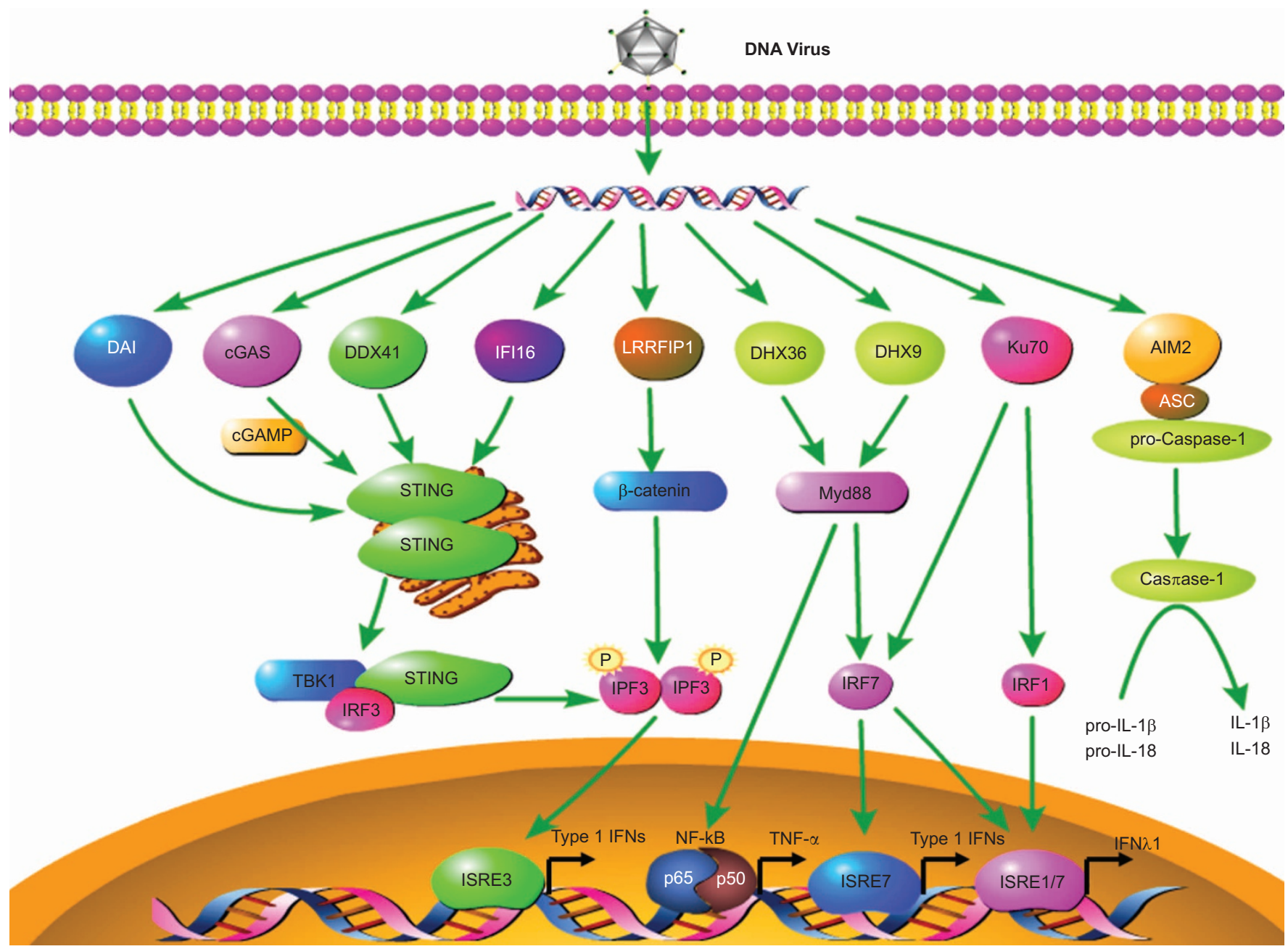

Figure 1 DNA sensing in the cytoplasm: recognition of cytosolic DNA appears to involve several sensors. DAI, DDX41, IFI16 or cGAS bind various cytosolic dsDNA, associate with STING and then activate the TBK1/IRF3 pathway, leading to the production of type I IFNs. AIM2 is a STING-independent cytosolic DNA sensor that forms the inflammasome with ASC to trigger caspase-1 activation and secretion of the pro-inflammatory cytokines IL-1 $\beta$ and IL18. DHX36 and DHX9 are identified as STING-independent but MyD88-dependent sensors of CpG-containing DNA in plasmacytoid dendritic cells. Another cytosolic dsDNA sensor, LRRFIP1, triggers IRF3-dependent production of IFN- $\beta$ in a $\beta$-catenin-dependent manner. Ku70 also functions independently of STING to induce the production of types I and III IFN. AIM2, absent in melanoma 2; cGAS, cGAMP synthase; DAI, DNA-dependent activator of interferon-regulatory factor; IFN, interferon; LRRFIP1, leucine-rich repeat (in Flightless I) interacting protein-1.

However, the essential function of AIM2 in DNA-mediated cleavage of pro-IL-1 $\beta$ has been confirmed. ${ }^{5,12}$ The authors further clarified whether Rad50-CARD9 induces the production of IL- $1 \beta$ through modulation of the inflammasome. They found that DNA-stimulated activation of caspase-1 was normal in CARD9-deficient DCs; these results indicate the mechanism for DNA-induced generation of IL-1 $\beta$, in which CARD9 complexes mediate the generation of pro-IL-1 $\beta$, which is subsequently processed by the AIM2 inflammasome. ${ }^{3}$

Finally, they confirmed the roles of Rad50, CARD9 and Bcl-10 during viral infection. After infection with vaccinia virus, the generation of IL- $1 \beta$ and other cytokines (IL-6 and TNF) was CARD9and Bcl-10-dependent, and foci of DNARad50-CARD9 complexes were visible in the cytoplasm. After in vivo intravenous infection with vaccinia virus, CARD9-deficient mice showed diminished IL- $1 \beta$ production and significantly impaired $\mathrm{CD}^{+}{ }^{+}$-cell responses compared to wild-type mice.

Roth et $a l .{ }^{4}$ demonstrate the existence of a novel cytosolic DNA sensing mechanism that induces IL- $1 \beta$ production (Figure 2). Transfection of DCs with dsDNA or infection of DCs with a DNA virus induced the formation of dsDNARad50-CARD9 signaling complexes, and this novel complex controlled NF- $\kappa \mathrm{B}$ activation and pro-IL-1 $\beta$ transcription in a cGAS-STING-independent manner. Although STING is essential for DNAtriggered innate immune responses and is thought to contribute to optimal NF$\kappa \mathrm{B}$ responses, it had no effect on the generation of IL-1 $\beta$ in this pathway. Nonetheless, the role of STING in DNA-mediated activation of NF- $\kappa B$ still requires further investigation.

Rad50, together with Mre11 and Nbs1 (MRN complex), plays a central role in double-strand break repair and DNA 


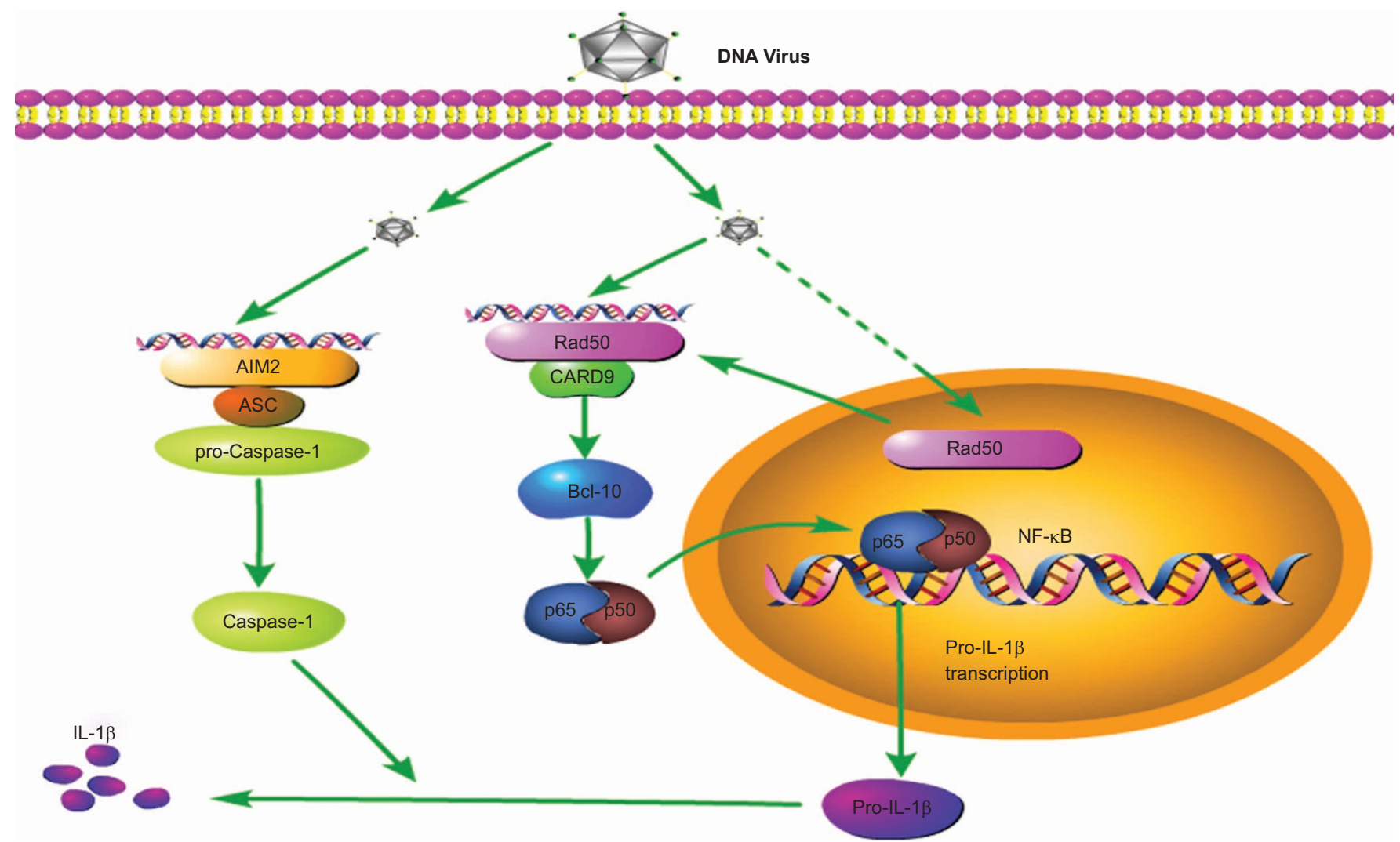

Figure 2 Cytosolic DNA recognition pathway for IL-1 $\beta$ production. Upon recognition of DNA, AIM2 interacts with ASC, a common adaptor of the inflammasome, leading to the assembly of an inflammasome and the cleavage of caspase-1, which induces the processing of pro-IL-1 $\beta$ and the secretion of IL-1 $\beta$. Roth et al. ${ }^{4}$ demonstrated a new cytosolic DNA sensing pathway that was able to induce IL-1 $\beta$. The presence of DNA or a DNA virus in the cytosol induces the formation of dsDNA-Rad50-CARD9 signaling complexes, which control NF- $\kappa$ B activation and the transcription of the gene encoding pro-IL-1 $\beta$. AIM2, absent in melanoma 2; CARD9, caspase recruitment domain-containing protein 9.

recombination in the nucleus. In this paper, the authors found that Rad50 translocates to the cytoplasm to recognize viral DNA. These results demonstrate crosstalk between the DNA-damage response system and the innate immune response to pathogens. However, a series of issues are raised by these findings. What signals Rad50 to move out of the nucleus and sense cytosolic DNA? How do the three members of the MRN complex cooperate during the relocation and DNA sensing process? The communication between nuclear proteins and cytosolic DNA requires further investigation.

IL-1 $\beta$ is a crucial pro-inflammatory cytokine that needs to be tightly controlled, as its aberrant generation can induce severe pathological conditions. IL- $1 \beta$ is not only important for immunological defense, but is also an important factor in inflammatory autoimmune diseases. ${ }^{13}$ The findings in this paper indicate that the new Rad50/CARD9/ Bcl-10 pathway may be expected to contribute to auto-inflammatory conditions associated with responses to cytosolic selfDNA. Collectively, Roth et al. ${ }^{4}$ define a cytosolic DNA recognition pathway for inflammation and a physical and functional connection between a conserved DNA damage sensor and the innate immune response to pathogens. Further studies of the Rad50/CARD9/Bcl-10 pathway may benefit mechanistic studies and therapeutic design for autoimmune and cytosolic DNA-induced inflammatory diseases.

1 Paludan SR, Bowie AG. Immune sensing of DNA. Immunity 2013; 38: 870-880.

2 Sun L, Wu J, Du F, Chen X, Chen ZJ. Cyclic GMP-AMP synthase is a cytosolic DNA sensor that activates the type I interferon pathway. Science 2013; 339: 786-791.

3 Yang $\mathrm{P}$, An $\mathrm{H}$, Liu X, Wen M, Zheng Y, Rui Y et al. The cytosolic nucleic acid sensor
LRRFIP1 mediates the production of type I interferon via a beta-catenin-dependent pathway. Nat Immunol 2010; 11: 487494.

4 Roth S, Rottach A, Lotz-Havla AS, Laux V, Muschaweckh A, Gersting SW et al. Rad50CARD9 interactions link cytosolic DNA sensing to IL-1beta production. Nat Immunol 2014; 15: 538-545.

5 Rathinam VA, Jiang Z, Waggoner SN, Sharma $S$, Cole LE, Waggoner $L$ et al. The AIM2 inflammasome is essential for host defense against cytosolic bacteria and DNA viruses. Nat Immunol 2010; 11: 395-402.

6 Gross 0, Gewies A, Finger K, Schafer M, Sparwasser T, Peschel C et al. Card9 controls a non-TLR signalling pathway for innate anti-fungal immunity. Nature 2006; 442: 651-656.

7 Hara H, Ishihara C, Takeuchi A, Imanishi T, Xue L, Morris SW et al. The adaptor protein CARD9 is essential for the activation of myeloid cells through ITAM-associated and Toll-like receptors. Nat Immunol 2007; 8: 619-629.

8 Hsu YM, Zhang Y, You Y, Wang D, Li H, Duramad $O$ et al. The adaptor protein CARD9 is required for innate immune responses to intracellular pathogens. Nat Immunol 2007; 8: 198-205. 
9 Poeck H, Bscheider M, Gross O, Finger K, Roth S, Rebsamen $\mathrm{M}$ et al. Recognition of RNA virus by RIG-I results in activation of CARD9 and inflammasome signaling for interleukin 1 beta production. Nat Immunol 2010; 11: 63-69.

10 Strasser D, Neumann K, Bergmann H, Marakalala MJ, Guler R, Rojowska A et al. Syk kinase-coupled C-type lectin receptors engage protein kinase $\mathrm{C}$ sigma to elicit Card9 adaptor-mediated innate immunity. Immunity 2012; 36: 32-42.

11 Stracker TH, Petrini JH. The MRE11 complex: starting from the ends. Nat Rev Mol Cell Biol 2011; 12: 90-103.

12 Fernandes-Alnemri T, Yu JW, Juliana C, Solorzano L, Kang S, Wu J et al. The
AlM2 inflammasome is critical for innate immunity to Francisella tularensis. Nat Immunol 2010; 11: 385393.

13 Dinarello CA, Simon A, van der Meer JW. Treating inflammation by blocking interleukin-1 in a broad spectrum of diseases. Nat Rev Drug Discov 2012; 11 633-652. 